\section{Clinical and genetic aspects of bicuspid aortic valve: a proposed model for family screening based on a review of literature}

\author{
Hubert Baars, ${ }^{1-3}$ Eline Overwater, ${ }^{4,5}$ \\ Marieke Baars, ${ }^{4}$ Barbara Mulder, ${ }^{6}$ \\ Wilhelmina Kerstjens-Frederikse, ${ }^{7}$ \\ Klaartje van Engelen, ${ }^{5}$ Arjan Houweling ${ }^{5}$ \\ 'Department of Cardiology, Bergman \\ Clinics, Bilthoven; ${ }^{2}$ Department of \\ Cardiology, University Medical Center \\ Utrecht, Utrecht; ${ }^{3}$ Department of \\ Cardiology, TweeSteden Hospital, \\ Tilburg; ${ }^{4}$ Department of Clinical Genetics, \\ Academic Medical Center, Amsterdam; \\ ${ }^{5}$ Department of Clinical Genetics, VU \\ University Medical Center, Amsterdam; \\ 'Department of Cardiology, Academic \\ Medical Center, Amsterdam; ${ }^{7}$ Department \\ of Genetics, University of Groningen, \\ University Medical Center Groningen, \\ Groningen, The Netherlands
}

\section{Abstract}

Bicuspid aortic valve (BAV) is the most common congenital cardiac defect causing serious morbidity including valvular dysfunction and thoracic aortic aneurysms (TAA) in around $30 \%$ of BAV patients. Cardiological screening of first-degree relatives is advised in recent guidelines given the observed familial clustering of BAV. However, guidelines regarding screening of family members and DNA testing are not unequivocal. The aim of this review is to provide an overview of the literature on echocardiographic screening in first-degree relatives of BAV patients and to propose a model for family screening. In addition, we provide a flowchart for DNA testing. We performed a PubMed search and included studies providing data on echocardiographic screening in asymptomatic relatives of BAV patients. Nine studies were included. In $5.8-47.4 \%$ of the families BAV was shown to be familial. of the screened first-degree relatives $1.8-11 \%$ was found to be affected with BAV. Results regarding a potential risk of TAA in first-degree relatives with a tricuspid aortic valve (TAV) were conflicting. The reported familial clustering of BAV underlines the importance of cardiological screening in relatives. After reviewing the available family history, patient characteristics and the results of cardiological screening in relatives, follow-up in relatives with a TAV and/or DNA testing may be advised in a subset of families. In this study we propose a model for the clinical and genetic work-up in BAV families, based on the most extensive literature review on family screening performed until now.

\section{Introduction}

With an estimated prevalence of $0.5-2 \%$ bicuspid aortic valve (BAV) (OMIM\#109730) is the most common congenital cardiac defect associated with an increased risk of serious complications, including thoracic aortic aneurysms (TAA). ${ }^{1-6}$ As BAV, either with or without associated TAA (+/-TAA), often is a familial condition, cardiological screening of first-degree relatives of $\mathrm{BAV}$ patients has been advised in recent guidelines of the American College of Cardiology and American Heart Association (ACC/AHA) ${ }^{7-9}$ However, screening is currently largely dependent on local initiatives and several important questions remain to be addressed. For example, it remains unclear whether relatives with a tricuspid aortic valve (TAV) are at an increased risk for the development of TAA and if DNA testing is a useful tool in the identification of families with a high risk for TAA at young age. The aim of this study is to provide an overview of the literature on family screening in firs-degree relatives, to provide an overview of the results of DNA testing in BAV (+/-TAA) families and to propose a model for clinical and genetic workup in BAV (+/-TAA) families. The results of the literature search and our proposed screening model are presented after a general overview of the clinical and genetic aspects of BAV $(+/-$ TAA). The current understanding of the pathology, clinical aspects and management of BAV disease and genetic syndromes associated with BAV were recently reviewed and are outside the scope of our study. ${ }^{10-13}$

\section{Bicuspid aortic valve: clinical and genetic aspects}

The bicuspid aortic valve, in most cases, consists of two unequal sized leaflets. The larger leaflet typically has a central raphe or ridge resulting from a fusion of the commissures (in $\sim 70 \%$ fusion of the right and left coronary cusp, the remainder mostly from fusion of the right and non coronary cusp, and rarely the left and non coronary cusp) resulting in a functionally bicuspid aortic valve. ${ }^{10,14} \mathrm{~A}$ central raphe is absent in the less frequently occurring true bicuspid valve. ${ }^{15-17}$ BAV can be an isolated congenital anomaly, but can also be associated with other abnormalities such as aortic coarctation, ventricular septal defects
Correspondence: Hubert F. Baars, Bergman Clinics Bilthoven, Prof. Bronkhorstlaan 10, 3723 MB Bilthoven, The Netherlands.

Tel.: 088.9000500. E-mail: h.baars@bergmanclinics.nl

Key words: bicuspid aortic valve, thoracic aortic aneurysm, genetics, family screening.

Conflict of interest: the authors declare no potential conflict of interest.

Contributions: HB, EO, KvE, AH, contributed equally. All authors made substantial contributions to the design, drafting and approval of the manuscript. All authors agree to be accountable for the manuscript.

Received for publication: 26 November 2014 Accepted for publication: 21 January 2015.

This work is licensed under a Creative Commons Attribution NonCommercial 3.0 License (CC BYNC 3.0).

CCopyright H. Baars et al., 2015

Licensee PAGEPress, Italy

Cardiogenetics 2015; 5:4842

doi:10.4081/cardiogenetics.2015.4842

and hypoplastic left ventricle. ${ }^{6,9,12}$

In most cases, BAV can be diagnosed and hemodynamically assessed using transthoracic echocardiography. In a minority of patients, particularly in calcific valve disease, higher resolution imaging techniques such as cardiac magnetic resonance imaging, computed tomography or trans-esophageal echocardiography may be required. ${ }^{16,18,19}$ Although BAV may retain normal function throughout adult life, around $30 \%$ of the people with BAV will develop clinical complications..$^{3-6}$ Therefore patients with BAV are advised to remain under regular surveillance by a cardiologist. ${ }^{9}$ Taking into account the high incidence, BAV may be responsible for more morbidity and mortality than other congenital cardiac defects combined. ${ }^{5,6}$ In patients with BAV aortic stenosis and/or insufficiency is more frequent. In addition, BAV patients are more prone to aortic dilatation. ${ }^{16}$ Dilatation of the thoracic aorta, most commonly located in the ascending aorta, has been reported in $35-80 \%$ of adult BAV patients and has rarely been observed as early

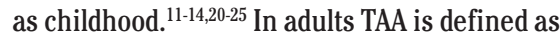
an aortic diameter with a z-score of $\geq 2$, corresponding to an observed value $>1.96$ standard deviations above the predicted value for age, gender, and body surface area (BSA).$^{26} \mathrm{An}$ aortic root $>4.0 \mathrm{~cm}$ in adults is considered to be dilated irrespective of age, gender or BSA. ${ }^{27-29}$ Aortic dilatation in BAV may be restricted to the ascending aorta, but can also include the aortic root. ${ }^{25,30}$ In a retrospective study among $241 \mathrm{BAV}$ patients referred for cardiac surgery, 
mean age 56 (range 16-85) years, aortic dilatation was seen in 97 patients (40\%). The aortic root was involved in $9(9.3 \%)$, the ascending aorta in $68(70.1 \%)$, both the root and the ascending aorta in 14 (14.4\%), the ascending aorta and the aortic arch in 5 (5.2\%) and the root, ascending and aortic arch in 1 of the patients (1\%). ${ }^{25}$ The relatively rare dilatation restricted to the aortic root is most commonly observed in men below the age of 40 and is reported to be associated with an increased risk of aortic dissection. This root phenotype has been proposed to be the form of bicuspid aortopathy most likely to be associated with a genetic cause. ${ }^{3,13,20,24,31-33}$

Although rare, the most feared complication in BAV patients is thoracic aortic dissection, which has been reported at young age. ${ }^{34}$ Whereas the lifetime risk of aortic dissection in BAV patients was initially reported around $5 \%$, recent studies show a lifetime risk of aortic dissection of less than $1 \%$ in BAV patients and a normal life expectancy. ${ }^{4,6,35,36}$ This difference can potentially be explained by increased surveillance and timely surgical intervention in recent years. Aortic dissection is, in the majority of cases, preceded by slowly progressive asymptomatic aortic dilatation. This allows screening and preventive surgery when indicated. Surgery is recommended in BAV patients with a diameter of the aortic sinuses or ascending aorta $>5.5 \mathrm{~cm}$, or $>5.0 \mathrm{~cm}$ in the presence of an additional risk factor (growth $\geq 0.5 \mathrm{~cm} /$ year or a family history of aortic dissection) ${ }^{37,38}$ Although the risk of aortic dissection is lower than initially estimated, cardiovascular surgery was performed in $22-27 \%$ of the BAV patients during follow-up.,

Familial BAV associated with dilatation of the aorta was first reported in literature over 40 years ago with the observation of a BAV in both a father and his son, who died in his sleep at the age of 19. No features of Marfan syndrome were observed. Autopsy confirmed an aortic dissection and a BAV. ${ }^{39}$ The first systematic study on cardiological screening in asymptomatic relatives of BAV patients, to our knowledge, was published in $1978 .{ }^{40}$ The authors screened 188 first-degree relatives of $41 \mathrm{BAV}$ patients by auscultation and eccentricity index. BAV was diagnosed in $3.7-9.6 \%$ of the relatives and was familial in $14.6-31.7 \%$ of the 41 families (depending on the inclusion of doubtful cases). The familial clustering of BAV (+/-TAA) has since been confirmed by a number of studies, indicating a high heritability of BAV $^{41}$ In addition, $20 \%$ of pediatric patients with a left ventricular outflow tract obstruction have (an) affected first-degree relative(s), frequently a previously undetected BAV further illustrating a strong genetic contribution to the origin of $\mathrm{BAV}^{42}$ However, to date, a genetic cause has been identified in only a minority of BAV families, mostly showing an autosomal dominant inheritance pattern with reduced penetrance. In 2005 NOTCH1 mutations were reported to be involved in a spectrum of developmental aortic valve anomalies, including BAV and severe aortic valve calcification. ${ }^{43}$ Sequence analysis of NOTCH1 indicated a potential overrepresentation of non-synonymous missense variants among BAV (+/-TAA) patients, however NOTCH1 mutations were found only in $<5 \%$ of BAV cases in several subsequent studies. ${ }^{43-49}$ Interestingly, NOTCH1 mutations were recently shown to cause Adams Oliver syndrome. This is a rare developmental disorder with aplasia cutis of the scalp and transverse limb defects, frequently associated with cardiac defects, including BAV. ${ }^{50}$ Based on studies in individual patients, linkage analysis in families and animal studies, several other genes and candidate loci have been implicated to be potentially involved in BAV. ${ }^{51-58}$ These studies emphasize the genetic as well as phenotypic heterogeneity of BAV.

Whether dilatation of the proximal aorta in patients with BAV is a primary manifestation of an underlying genetic disorder, or secondary to hemodynamic effects related to the abnormal aortic valve remains controversial. . $1,32,59,60$ Martin et al. performed bivariate genetic analyses between aortic dimensions and BAV. Their results did not support a shared underlying genetic basis for BAV and aortic measures. ${ }^{60}$ However, Loscalzo et al. conclude that BAV and TAA might be independent manifestations of a single gene defect with an autosomal dominant pattern of inheritance with incomplete penetrance. They studied segregation of BAV and TAA in 13 TAA families referred for analysis of known aneurysm, dissection or rupture. In total 110 first-degree relatives of index patients were included. In 15 (13.6\%) BAV was seen, in 10 cases associated with TAA. Twenty four of the relatives (22\%) were diagnosed with TAA in the presence of a normal tricuspid aortic valve. ${ }^{32}$ In addition, Keane et al. reported that aortic size in BAV patients was larger than in control patients. They observed comparable degrees of aortic regurgitation, stenosis or mixed lesions, and concluded that intrinsic pathology appears to be responsible for aortic enlargement. ${ }^{21}$ These observations support the hypothesis that BAV and TAA are independent manifestations of a single gene defect in a subset of BAV families..$^{21,32,61}$ The risk of dilatation of the thoracic aorta might therefore be increased in some first-degree relatives of BAV patients, even in the absence of a bicuspid aortic valve. Vice versa, a limited number of studies suggest that BAV might be more prevalent in familial thoracic aneurysms and dissections (FTAAD) and in some hereditary connective tissue disorders associated with an increased risk of aortic aneurysms (for example Marfan syndrome). ${ }^{33,62-64}$ Several studies illustrate the value of DNA testing in fami- lies with BAV +/- TAA by the identification of pathogenic mutations in known TAAD genes in FTAAD families with affected individuals with BAV. This provides further evidence for the hypothesis that both TAA and BAV may be independent phenotypic manifestations of a single mutation. ${ }^{31,33,62}$ BAV was identified in four FTAAD patients in three of 14 studied families with an autosomal dominant pattern of thoracic aortic dissections on the basis of a mutation in smooth muscle aortic actin (ACTA2) gene. ${ }^{62}$ A mutation in transforming growth factor- receptor type II (TGFBR2) gene, associated with Loeys Dietz syndrome, was found in a 48-year-old woman with BAV and a proximal aortic aneurysm including the aortic root, measuring $56 \mathrm{~mm}$. Her brother died suddenly at the age of 42 immediately after the onset of excruciating chest pain. Her father, who also carried the mutation, had a history of elective surgical replacement of the aortic valve and ascending aorta for BAV and ascending aortic aneurysm at the age of $72 .{ }^{33}$ In several subsequent studies no TGFBR1 or TGFBR2 mutations were found in sporadic and familial BAV. $^{32,44,64}$ Recently, FBN1-mutations were linked to $\mathrm{BAV}$ as well by the identification of three FBN1 mutations in two BAV patients with TAA (age at diagnosis 15 and 19 years) who did not fulfill the Marfan syndrome criteria according to the revised Ghent criteria. ${ }^{64}$ In both patients the aortic diameter exceeded the threshold for surgery and the aortic size was largest at the level at the sinuses of Valsalva. Two of the three mutations (pArg529Gln and Arg2726Trp) were previously identified to be associated with variable/incomplete Marfan phenotype. In addition, the prevalence of BAV was reported to be increased in a cohort of 257 unrelated patients diagnosed with Marfan syndrome according to the updated Ghent criteria. Echocardiography showed BAV in 12 patients (4.7\%). ${ }^{63}$ In three of these 12 patients DNA testing was performed revealing a pathogenic FBN1 mutation in two cases, supporting the hypothesis that FBN1 mutations may not only be associated with an increased incidence TAA, but also with BAV.

\section{Overview of the literature on family screening in first-degree relatives of bicuspid aortic valve patients}

We performed a PubMed search using the term bicuspid aortic valve in combination with at least one of the following terms in title and/or abstract: gene(s), genetic(s), syndrome(s), family, relatives, family screening, pedigree analysis, inherited, aortic aneurysm, aortic dilatation or aortic dissection. Inclusion 
criteria were: studies providing data on systematic cardiological screening using echocardiography in first degree relatives of at least 20 BAV index patients, English language, published before November 2014. The number of at least 20 probands was arbitrarily chosen as we estimated that smaller cohorts or case series might introduce uncontrollable bias.

Using the abovementioned search strategy, we identified 683 articles. These articles were assessed for eligibility by reading title, abstract and/or full text. Of these, 27 contained data on family screening. Nine studies met our inclusion criteria. The results of these studies are

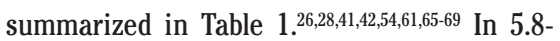
$47.7 \%$ of the families BAV was shown to be familial (defined as BAV diagnosed in at least one first degree relative of the index patient). Of the screened first-degree relatives of BAV patients $1.8-11 \%$ was found to be affected with $\mathrm{BAV}$. The results regarding the risk of TAA with $\mathrm{TAV}$ in first degree relatives are conflicting. One study reported the presence of TAA in $32 \%$ of first degree relatives with $\mathrm{TAV}^{61}$ In this study, $53 \%$ of the BAV index patients had a dilated aortic root. Dilatation of the aortic root is described to be relatively rare in BAV and is proposed to be the form of bicuspid aortopathy most likely to be associated with a genetic

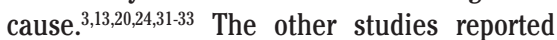
percentages of TAA in the first-degree TAV relatives of $3-4 \% .{ }^{54,65-68}$ This is around population risk since $2.3 \%$ of the general population, by definition, is expected to have $\mathrm{z}$ scores $>2 .{ }^{28,70,71}$ In the large study by RobledoCarmona et al. only mild aortic dilatation $(<4$ $\mathrm{cm}$ ) at older age ( $>50$ years), was observed among nine out of 270 first degree relatives with a TAV, comparable to the observations in the control cohort. ${ }^{6}$ They concluded that if their findings are confirmed by other studies, echocardiographic follow up of the aortic dimensions of TAV first-degree relatives might not be necessary. ${ }^{68}$ In addition, in a recent study by Dayan et al among first-degree relatives of BAV patients without TAA, normal aortic dimensions were seen in all relatives with a tricuspid valve. ${ }^{72}$

\section{A proposed model for clinical and genetic work-up in bicus- pid aortic valve (+/-TAA) fam- ilies based on literature}

Based on our review of the literature and our clinical practice, we propose the model shown in Figure 1 as a tool for the clinical and genetic screening of BAV patients and their relatives. The main purpose of this model is identifying at risk relatives for BAV and TAA. Following the ACC/AHA guidelines and the rec- ommendations of the authors of the nine studies on family screening, we recommend cardiological screening including echocardiography in all first degree relatives ( $>18$ years) ${ }^{7-9} \mathrm{We}$ do not perform screening in asymptomatic children given the low chance of significant abnormalities not detected during the routinely performed prenatal ultrasound at 20 weeks gestation and cardiac auscultation after birth. After reviewing the available family history, patient characteristics and the results of cardiological screening in relatives, follow-up in relatives with a TAV and/or DNA testing may be advised (Figure 1). In case of sporadic or familial cases of BAV without TAA or aortic dissection in relatives, we do not recommend follow up in first-degree relatives with a tricuspid valve and normal aortic diameters. In these families, we only recommend DNA diagnostics in the index patient and echocardiographic follow up in the first-degree relatives (e.g. every five years, depending on the age and echocardiographic findings) when dilatation of the aortic root in the BAV index patient develops before the age of 60 years. Based on our review of literature, with most studies showing only a slightly increased risk of TAA in relatives with $\mathrm{TAV}$, we advise clinical follow up of first-degree relatives with TAV only in the following situations; sporadic BAV with aortic root involvement before the age of 60 years, TAA in a relative with TAV before the age of 60 , and in families with two or more persons with (suspected) TAA and a TAV irrespective of age. The cut off at the age of 60 years is more or less arbitrary and is chosen since increased aortic diameters are more likely to be associated with older age and hypertension then with genetic factors when observed later in life. ${ }^{73}$ In contrast, aortic root involvement at young age is relatively rare in $\mathrm{BAV}$, but relatively frequent in FTAAD and syndrome associated TAAD, and might be associated with mutations in TAAD genes. ${ }^{33,62,64,74,75}$ First-degree relatives of young BAV patients with dilatation of the aortic root may therefore have an increased risk of TAA in absence of BAV. ${ }^{22,32,61,62}$ In our clinical genetics department DNA diagnostics of 13 TAAD genes (ACTA2, COL3A1, EFEMP2, ELN, FBN1, FBN2, MYH11, MYLK, PLOD1, SLC2A10, SMAD3, TGFBR1, TGFBR2) and NOTCH1 is offered in the following three situations: BAV patients with aortic root dilatation ( $\mathrm{z}>2$ or an aortic diameter $>4.0 \mathrm{~cm}$ ) before the age of 60 , in case of TAA in a relative with TAV before the age of 60 years, and in familial TAA. Furthermore, in confirmed familial BAV cases not fulfilling the aforementioned criteria, sequencing of the NOTCH1 gene may be considered. In sporadic cases of BAV, or sporadic BAV patients with TAA $<60$ years not involving the aortic root, the chance of identifying a pathogenic mutation in NOTCH1 is likely to be low. NOTCH1 mutation screening in these patients may be considered, depending on the social context of the patient, but is not routinely recommended by us (Figure 1). In familial BAV without TAA, NOTCH1 mutation screening can be helpful to identify at risk relatives, especially in patients with calcified bicuspid valves. ${ }^{43}$ We currently advise follow-up of all relatives carrying a pathogenic NOTCH1 mutation irrespective of the presence or absence of BAV given a potentially increased risk of TAA. ${ }^{48}$ Future phenotypegenotype studies may potentially enable the identification of specific NOTCH1 mutations associated with an increased risk for TAA. When using our proposed model it is important to consider all available clinical data of a family in its entirety. In case of new information during follow-up one should reconsider the situation using the model and adjust the clinical and genetic work-up accordingly (for example in case of the development of an aortic root aneurysm in a BAV patient or a newly diagnosed family member with BAV). This model is meant as a tool for non-syndromic BAV $(+/-$ TAA) patients and their families. In case of evidence for a hereditary connective tissue disorder we recommend a custom multidisciplinary work-up and targeted analysis of candidate genes for the suspected underlying syndrome instead of using the presented model. For example in cases of extreme degrees of aortic dilatation, and/or the presence of other symptoms or features. The individual work-up in patients with (suspected) genetic syndromes associated with an increased occurrence of BAV, such as Turner syndrome, is outside the scope of our study.

\section{Discussion and Conclusions}

We propose a model for the clinical and genetic screening in non-syndromic BAV (+/TAA) families based on a review of the literature. The percentage of BAV in first-degree relatives of BAV index patients was $1.8-11 \%$ and BAV was found to be familial in $5.8-47.4 \%$ of the families in the nine included studies. The different percentages reported in literature might be explained by the small number of index patients and screened first-degree relatives in some studies. Furthermore, the participation rates of the first-degree relatives in cardiological screening varied between studies and were not mentioned in all papers. This could have resulted in a selection bias. In addition, different patient groups with potentially different prevalences of BAV were included (e.g. pediatric patients with/without additional congenital cardiac defects versus adult index patients). Furthermore, the prevalence of (familial) BAV might differ between geographical regions as suggested by Robledo-Carmona et al. ${ }^{68}$ In all studies, the importance of screen- 


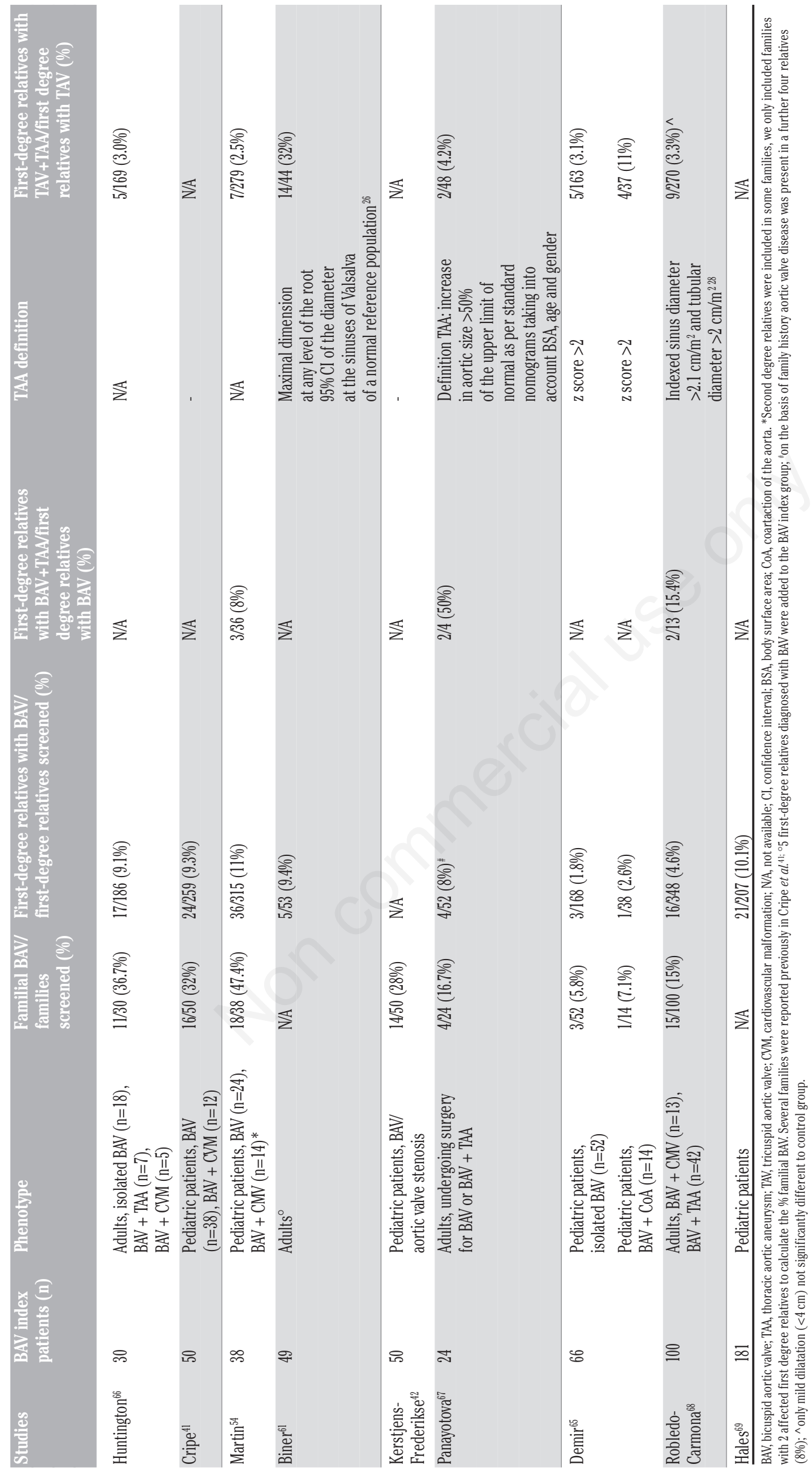




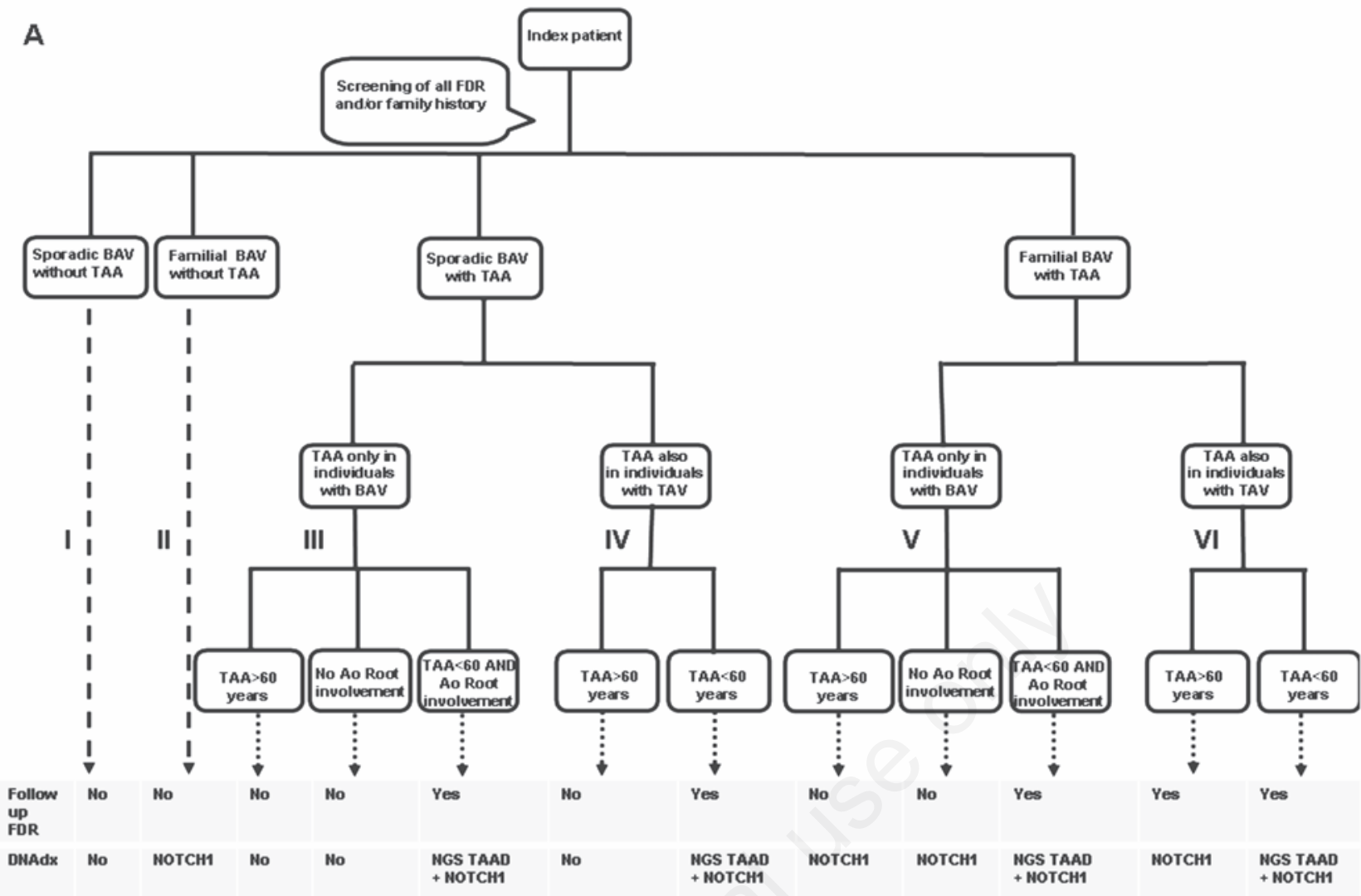

B

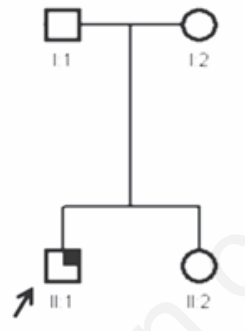

Sporadic BAV without TAA

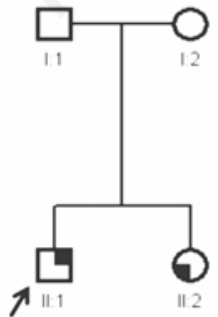

Sporadic BAV with TAA:

TAA also in individual with TAV

IV

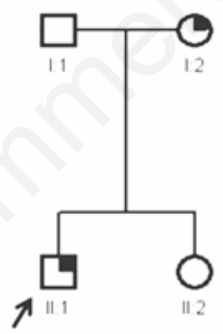

Familial BAV without TAA

II

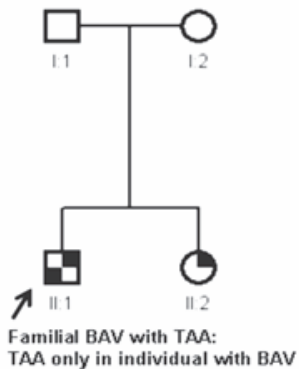

V

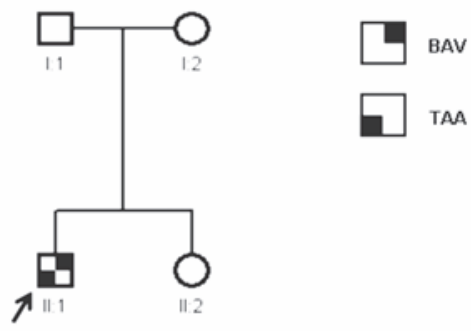

Sporadic BAV with TAA:

TAA only in individual with BAV

III

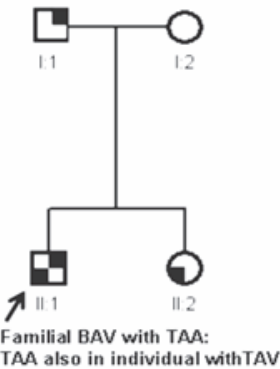

VI

Figure 1. A) Flow chart illustrating a proposed model for genetic testing, and clinical work-up of first-degree relatives (FDR) of patients with bicuspid aortic valve (BAV). Clinical follow up of first-degree relatives comprises echocardiographic screening at a low frequency (e.g., every five years) starting at the age of 18 years. In our center NGS TAAD analysis includes: ACTA2, COL3A1, EFEMP2, ELN, FBN1, FBN2, MYH11, MYLK, PLOD1, SLC2A10, SMAD3, TGFBR1 and TGFBR2. B) Simplified pedigrees illustrating examples of the different familial situations in the flow chart. Men are encoded with squares, women with circles. Index patients are indicated with an arrow. Ao root involvement, aortic root involvement; NGS, next generation sequencing; TAA, thoracic aortic aneurysm; TAV, tricuspid aortic valve. 
ing in first-degree relatives of BAV patients was underlined by the authors. One study addressed the cost-effectiveness of cardiological screening in siblings of children with BAV. It was concluded that screening is effective and inexpensive and should be incorporated into clinical care. ${ }^{69}$ We feel confident that, using our model, the majority of high-risk families can be identified. In our opinion, repeated follow up in all first-degree relatives of all BAV patients poses an unjustifiable burden on health resources. Although based on a few studies only, DNA sequencing appears to be a promising tool in the identification of a subset of high-risk families. Aortic dissections at a young age are suggestive for a connective tissue disorder or FTAAD, whereas dissections in BAV patients usually occur later in life. The mean age at presentation (either detection of an aneurysm or after presentation with a complication) was lowest in syndrome associated and monogenic aneurysm patients at around 25-27 years versus $55-57$ years in familial aneurysms and 64-66 years in the sporadic aneurysm group, and 55 years in the BAV group. ${ }^{76}$ However, the ages at presentation within these groups were highly variable and largely overlapping, further complicating differentiating between sporadic BAV, familial BAV (+/-TAA), FTAAD and syndromal TAAD when faced with an individual patient without screening of relatives. ${ }^{75-78}$ The reported location of aortic dilatation in the families with an identifiable pathogenic mutation in FBN1, $A C T A 2$ or TGFBR2 included the aortic root and dissections were observed at young age. Mutations in these genes are associated with a high risk of aortic dilatation and dissection at young age, in most cases in patients with tricuspid aortic valves. Although evaluation in larger cohorts is required, DNA diagnostics appears to be a promising and valuable tool in identifying a minority of high-risk families presenting with an index patient with BAV (+/TAA). The identification of a pathogenic mutation enables genetic testing of family members and selective clinical follow up of at risk relatives. Furthermore, DNA testing is becoming widely available at rapidly declining costs and is increasingly incorporated into standard clinical practice. ${ }^{74,75}$ Novel techniques such as whole exome- and whole genome sequencing are likely to be valuable in the identification of novel genes in BAV and TAA. Therefore, it is likely that DNA testing will take a more prominent part in risk stratification in BAV (+/-TAA) patients and their families in the near future. In the Netherlands, genetic counseling and DNA testing in patients with suspected inherited cardiovascular disease is performed mainly by clinical geneticists and genetic counselors working in multidisciplinary teams also including pediatric cardiologists, cardiologists and social workers specialized in cardiogenet- ic disorders. These outpatient clinics provide well-equipped setting for the coordination of family screening, collecting of clinical data and, when indicated, performing dysmorphologic examination and DNA testing. BAV patients can be referred to these outpatient clinics when there is an indication for DNA testing and/or clinical follow-up of first-degree relatives on the basis of our flowchart or when a genetic syndrome is suspected. Other reasons for referral can for example be questions about the inheritance of BAV, including potential implications for family members and/or (future) offspring. Further insight into the genetic and pathophysiological mechanisms leading to BAV and/or TAA is required to enable the identification of novel factors causing health risks in BAV patients and their relatives. These include aortic medial degeneration, vascular smooth muscle cell apoptosis and the interplay between BAV morphology, sheer stress and valvular dysfunction and TAA in BAV patients,

Limitations of our model include the limited number of studies, different patient groups and the absence of DNA testing in the majority of publications. When using our proposed model it is important to consider all available clinical data of a family in its entirety. This may be difficult due to loss of follow up of the index patient (e.g. after moving or due to non compliance) and because of difficulties to obtain all relevant medical records of relatives (e.g. because not all first degree relatives are informed by the index patient and/or not all relatives are participating in cardiological screening). Long term follow up in well-characterized BAV cohorts is required to test the feasibility, sensitivity and specificity of our model which should be adjusted accordingly when necessary.

\section{References}

1. Basso C, Boschello M, Perrone C, et al. An echocardiographic survey of primary school children for bicuspid aortic valve. Am J Cardiol 2004;93:661-3.

2. Gray GW, Salisbury DA, Gulino AM. Echocardiographic and color flow Doppler findings in military pilot applicants. Aviat Space Environ Med 1995;66:32-4.

3. Michelena HI, Desjardins VA, Avierinos JF, et al. Natural history of asymptomatic patients with normally functioning or minimally dysfunctional bicuspid aortic valve in the community. Circulation 2008; 117:2776-84.

4. Tzemos N, Therrien J, Yip J, et al. Outcomes in adults with bicuspid aortic valves. JAMA 2008;300:1317-25.

5. Vallely MP, Semsarian C, Bannon PG.
Management of the ascending aorta in patients with bicuspid aortic valve disease. Heart Lung Circ 2008;17:357-63.

6. Ward C. Clinical significance of the bicuspid aortic valve. Heart 2000;83:81-5.

7. Hiratzka LF, Bakris GL, Beckman JA, et al. 2010 ACCF/AHA/AATS/ACR/ASA/SCA/SCAV/ SIR/STS/SVM Guidelines for the diagnosis and management of patients with thoracic aortic disease. A Report of the American College of Cardiology Foundation/American Heart Association Task Force on Practice Guidelines, American Association for Thoracic Surgery, American College of Radiology,American Stroke Association, Society of Cardiovascular Anesthesiologists, Society for Cardiovascular Angiography and Interventions, Society of Interventional Radiology, Society of Thoracic Surgeons, and Society for Vascular Medicine. J Am Coll Cardiol 2010;55:e27e129.

8. Vahanian A, Alfieri 0, Andreotti F, et al. Guidelines on the management of valvular heart disease (version 2012). Eur Heart J 2012;33:2451-96.

9. Warnes CA, Williams RG, Bashore TM, et al. ACC/AHA 2008 guidelines for the management of adults with congenital heart disease: a report of the American College of Cardiology/American Heart Association Task Force on Practice Guidelines (Writing Committee to Develop Guidelines on the Management of Adults With Congenital Heart Disease.. Developed in Collaboration With the American Society of Echocardiography, Heart Rhythm Society, International Society for Adult Congenital Heart Disease, Society for Cardiovascular Angiography and Interventions, and Society of Thoracic Surgeons. J Am Coll Cardiol 2008;52:e143e263.

10. Abdulkareem N, Smelt J, Jahangiri M. Bicuspid aortic valve aortopathy: genetics, pathophysiology and medical therapy. Interact Cardiovasc Thorac Surg 2013;17:554-9.

11. Fedak PW, Verma S, David TE, et al. Clinical and pathophysiological implications of a bicuspid aortic valve. Circulation 2002;106:900-4.

12. Siu SC, Silversides CK. Bicuspid aortic valve disease. J Am Coll Cardiol 2010;55: 2789-800.

13. Verma S, Siu SC. Aortic dilatation in patients with bicuspid aortic valve. N Engl J Med 2014;370:1920-9.

14. Calloway TJ, Martin LJ, Zhang X, et al. Risk factors for aortic valve disease in bicuspid aortic valve: a family-based study. Am J Med Genet A 2011;155A:1015-20. 
15. Maskatia SA, Justino H, Ing FF, et al. Aortic valve morphology is associated with outcomes following balloon valvuloplasty for congenital aortic stenosis. Catheter Cardiovasc Interv 2013;81:90-5.

16. Michelena HI, Prakash SK, Della Corte A, et al. Bicuspid aortic valve: identifying knowledge gaps and rising to the challenge from the International Bicuspid Aortic Valve Consortium (BAVCon). Circulation 2014;129:2691-704.

17. Sabet HY, Edwards WD, Tazelaar HD, Daly RC. Congenitally bicuspid aortic valves: a surgical pathology study of 542 cases (1991 through 1996) and a literature review of 2,715 additional cases. Mayo Clin Proc 1999;74:14-26.

18. Lee SC, Ko SM, Song MG, et al. Morphological assessment of the aortic valve using coronary computed tomography angiography, cardiovascular magnetic resonance, and transthoracic echocardiography: comparison with intraoperative findings. Int $\mathrm{J}$ Cardiovasc Imaging 2012;28:33-44.

19. Malaisrie SC, Carr J, Mikati I, et al. Cardiac magnetic resonance imaging is more diagnostic than 2-dimensional echocardiography in determining the presence of bicuspid aortic valve. J Thorac Cardiovasc Surg 2012;144:370-6.

20. Della Corte A, Bancone C, Quarto C, et al. Predictors of ascending aortic dilatation with bicuspid aortic valve: a wide spectrum of disease expression. Eur J Cardiothorac Surg 2007;31:397-404.

21. Keane MG, Wiegers SE, Plappert T, et al. Bicuspid aortic valves are associated with aortic dilatation out of proportion to coexistent valvular lesions. Circulation 2000;102:III35-9.

22. Nistri S, Sorbo MD, Marin M, et al. Aortic root dilatation in young men with normally functioning bicuspid aortic valves. Heart 1999;82:19-22.

23. Novaro GM, Tiong IY, Pearce GL, et al. Features and predictors of ascending aortic dilatation in association with a congenital bicuspid aortic valve. Am J Cardiol 2003;92:99-101.

24. Tadros TM, Klein MD, Shapira OM. Ascending aortic dilatation associated with bicuspid aortic valve: pathophysiology, molecular biology, and clinical implications. Circulation 2009;119:880-90.

25. Yuan SM, Jing H, Lavee J. The bicuspid aortic valve and its relation to aortic dilation. Clinics (Sao Paulo) 2010;65:497-505.

26. Lang RM, Bierig M, Devereux RB, et al. Recommendations for chamber quantification: a report from the American Society of Echocardiography's Guidelines and Standards Committee and the Chamber Quantification Writing Group, developed in conjunction with the European Association of Echocardiography, a branch of the European Society of Cardiology. J Am Soc Echocardiogr 2005;18:1440-63.

27. Kinoshita N, Mimura J, Obayashi C, et al. Aortic root dilatation among young competitive athletes: echocardiographic screening of 1929 athletes between 15 and 34 years of age. Am Heart J 2000;139:7238.

28. Roman MJ, Devereux RB, Kramer-Fox R, 0'Loughlin J. Two-dimensional echocardiographic aortic root dimensions in normal children and adults. Am J Cardiol 1989;64:507-12.

29. Radonic T, de WP, Groenink M, et al. Critical appraisal of the revised Ghent criteria for diagnosis of Marfan syndrome. Clin Genet 2011;80:346-53.

30. Detaint D, Michelena HI, Nkomo VT, et al. Aortic dilatation patterns and rates in adults with bicuspid aortic valves: a comparative study with Marfan syndrome and degenerative aortopathy. Heart 2014;100:126-34.

31. Pepe G, Nistri S, Giusti B, et al. Identification of fibrillin 1 gene mutations in patients with bicuspid aortic valve (BAV) without Marfan syndrome. BMC Med Genet 2014;15:23.

32. Loscalzo ML, Goh DL, Loeys B, et al. Familial thoracic aortic dilation and bicommissural aortic valve: a prospective analysis of natural history and inheritance. Am J Med Genet A 2007;143A:19607.

33. Girdauskas E, Schulz S, Borger MA, et al. Transforming growth factor-b receptor type II mutation in a patient with bicuspid aortic valve disease and intraoperative aortic dissection. Ann Thorac Surg 2011;91:e70-e71.

34. Edwards WD, Leaf DS, Edwards JE. Dissecting aortic aneurysm associated with congenital bicuspid aortic valve. Circulation 1978;57:1022-5.

35. Larson EW, Edwards WD. Risk factors for aortic dissection: a necropsy study of 161 cases. Am J Cardiol 1984;53:849-55.

36. Michelena HI, Khanna AD, Mahoney D, et al. Incidence of aortic complications in patients with bicuspid aortic valves. JAMA 2011;306:1104-12.

37. Nishimura RA, Otto CM, Bonow R0, et al. 2014 AHA/ACC Guideline for the Management of Patients With Valvular Heart Disease: Executive Summary: A Report of the American College of Cardiology/American Heart Association Task Force on Practice Guidelines. Circulation 2014 [Epub ahead of print].

38. Shimada I, Rooney SJ, Pagano D, et al. Prediction of thoracic aortic aneurysm expansion: validation of formulae describ- ing growth. Ann Thorac Surg 1999;67:1968-70.

39. McKusick VA. Association of congenital bicuspid aortic valve and Erdheim's cystic medial necrosis. Lancet 1972;1:1026-7.

40. Emanuel R, Withers R, O'Brien K, et al. Congenitally bicuspid aortic valves. Clinicogenetic study of 41 families. $\mathrm{Br}$ Heart J 1978;40:1402-7.

41. Cripe L, Andelfinger G, Martin LJ, et al. Bicuspid aortic valve is heritable. $\mathrm{J}$ Am Coll Cardiol 2004;44:138-43.

42. Kerstjens-Frederikse WS, Du Marchie Sarvaas GJ, Ruiter JS, et al. Left ventricular outflow tract obstruction: should cardiac screening be offered to first-degree relatives? Heart 2011;97:1228-32.

43. Garg V, Muth AN, Ransom JF, et al. Mutations in NOTCH1 cause aortic valve disease. Nature 2005;437:270-4.

44. Foffa I, Ait Ali L, Panesi P, et al. Sequencing of NOTCH1, GATA5, TGFBR1 and TGFBR2 genes in familial cases of bicuspid aortic valve. BMC Med Genet 2013;14:44.

45. Garg V. Molecular genetics of aortic valve disease. Curr Opin Cardiol 2006;21:180-4.

46. Kent KC, Crenshaw ML, Goh DL, Dietz HC. Genotype-phenotype correlation in patients with bicuspid aortic valve and aneurysm. J Thorac Cardiovasc Surg 2013;146:158-65.

47. McBride KL, Riley MF, Zender GA, et al. NOTCH1 mutations in individuals with left ventricular outflow tract malformations reduce ligand-induced signaling. Hum Mol Genet 2008;17:2886-93.

48. McKellar SH, Tester DJ, Yagubyan M, et al Novel NOTCH1 mutations in patients with bicuspid aortic valve disease and thoracic aortic aneurysms. J Thorac Cardiovasc Surg 2007;134:290-6.

49. Mohamed SA, Aherrahrou Z, Liptau H, et al. Novel missense mutations (p.T596M and p.P1797H) in NOTCH1 in patients with bicuspid aortic valve. Biochem Biophys Res Commun 2006;345:1460-5.

50. Stittrich AB, Lehman A, Bodian DL, et al. Mutations in NOTCH1 cause Adams-Oliver syndrome. Am J Hum Genet 2014 [Epub ahead of print].

51. Beffagna G, Cecchetto A, Dal BL, et al. R25C mutation in the NKX2.5 gene in Italian patients affected with non-syndromic and syndromic congenital heart disease. J Cardiovasc Med (Hagerstown) 2013;14:582-6.

52. Biben C, Weber R, Kesteven S, et al. Cardiac septal and valvular dysmorphogenesis in mice heterozygous for mutations in the homeobox gene Nkx2-5. Circ Res 2000;87:888-95.

53. Lee TC, Zhao YD, Courtman DW, Stewart DJ. Abnormal aortic valve development in 
mice lacking endothelial nitric oxide synthase. Circulation 2000;101:2345-8.

54. Martin LJ, Ramachandran V, Cripe LH, et al. Evidence in favor of linkage to human chromosomal regions $18 \mathrm{q}, 5 \mathrm{q}$ and $13 \mathrm{q}$ for bicuspid aortic valve and associated cardiovascular malformations. Hum Genet 2007;121:275-84.

55. Martin LJ, Pilipenko V, Kaufman KM, et al. Whole exome sequencing for familial bicuspid aortic valve identifies putative variants. Circ Cardiovasc Genet 2014 [Epub ahead of print].

56. Padang R, Bagnall RD, Richmond DR, et al. Rare non-synonymous variations in the transcriptional activation domains of GATA5 in bicuspid aortic valve disease. J Mol Cell Cardiol 2012;53:277-81.

57. Tan HL, Glen E, Topf A, et al. Nonsynonymous variants in the SMAD6 gene predispose to congenital cardiovascular malformation. Hum Mutat 2012;33:720-7.

58. Thomas PS, Sridurongrit S, Ruiz-Lozano P, Kaartinen V. Deficient signaling via Alk2 (Acvr1) leads to bicuspid aortic valve development. PLoS One 2012;7:e35539.

59. Hinton RB. Bicuspid aortic valve and thoracic aortic aneurysm: three patient populations, two disease phenotypes, and one shared genotype. Cardiol Res Pract 2012;2012:926975.

60. Martin LJ, Hinton RB, Zhang X, et al. Aorta measurements are heritable and influenced by bicuspid aortic valve. Front Genet 2011;2:61.

61. Biner S, Rafique AM, Ray I, et al. Aortopathy is prevalent in relatives of bicuspid aortic valve patients. J Am Coll Cardiol 2009;53:2288-95.
62. Guo DC, Pannu H, Tran-Fadulu V, et al. Mutations in smooth muscle a-actin (ACTA2) lead to thoracic aortic aneurysms and dissections. Nat Genet 2007;39:148893.

63. Nistri S, Porciani MC, Attanasio M, et al. Association of Marfan syndrome and bicuspid aortic valve: frequency and outcome. Int J Cardiol 2012;155:324-5.

64. Pepe G, Nistri S, Giusti B, et al. Identification of fibrillin 1 gene mutations in patients with bicuspid aortic valve (BAV) without Marfan syndrome. BMC Med Genet 2014;15:23.

65. Demir F, Karadeniz C, Atalay S, et al. Screening of families of patients with leftsided cardiovascular anomalies. Pediatr Int 2013;55:555-60.

66. Huntington K, Hunter AG, Chan KL. A prospective study to assess the frequency of familial clustering of congenital bicuspid aortic valve. J Am Coll Cardiol 1997;30: 1809-12.

67. Panayotova R, Macnab A, Waterworth PD. A pilot project of familial screening in patients with bicuspid aortic valve disease. J Heart Valve Dis 2013;22:150-5.

68. Robledo-Carmona J, Rodriguez-Bailon I, Carrasco-Chinchilla F, et al. Hereditary patterns of bicuspid aortic valve in a hundred families. Int J Cardiol 2013;168: 34439.

69. Hales AR, Mahle WT. Echocardiography screening of siblings of children with bicuspid aortic valve. Pediatrics 2014 [Epub ahead of print].

70. Pettersen MD, Du W, Skeens ME, Humes RA. Regression equations for calculation of $\mathrm{z}$ scores of cardiac structures in a large cohort of healthy infants, children, and adolescents: an echocardiographic study. J Am Soc Echocardiogr 2008;21:922-34.

71. Warren AE, Boyd ML, O'Connell C, Dodds L. Dilatation of the ascending aorta in paediatric patients with bicuspid aortic valve: frequency, rate of progression and risk factors. Heart 2006;92:1496-500.

72. Dayan V, Parma G, Drever M, et al. Firstdegree relatives of bicuspid aortic valve patients with normal aortic dimensions do not carry an increased risk of aortic dilatation. Int J Cardiol 2014;172:518-9.

73. Devereux RB, de Simone G, Arnett DK, et al. Normal limits in relation to age, body size and gender of two-dimensional echocardiographic aortic root dimensions in persons $>/=15$ years of age. Am $\mathrm{J}$ Cardiol 2012;110:1189-94.

74. De Backer J, Campens L, De Paepe A. Genes in thoracic aortic aneurysms/dissections - do they matter? Ann Cardiothorac Surg 2013;2:73-82.

75. Milewicz DM, Regalado ES, Shendure J, et al. Successes and challenges of using whole exome sequencing to identify novel genes underlying an inherited predisposition for thoracic aortic aneurysms and acute aortic dissections. Trends Cardiovasc Med 2014;24:53-60.

76. Coady MA, Davies RR, Roberts M, et al. Familial patterns of thoracic aortic aneurysms. Arch Surg 1999;134:361-7.

77. Jondeau G, Boileau C. Genetics of thoracic aortic aneurysms. Curr Atheroscler Rep 2012;14:219-26.

78. Albornoz G, Coady MA, Roberts M, et al. Familial thoracic aortic aneurysms and dissections - incidence, modes of inheritance, and phenotypic patterns. Ann Thorac Surg 2006;82:1400-5. 